\title{
Der deutsche Venture-Capital-Markt - Investitionen und Rahmenbedingungen
}

\author{
Innovative Start-ups sind aus mehreren Gründen durch ein hohes Risiko gekennzeichnet. \\ Venture Capital (VC) ermöglicht es Start-ups, ihre Ideen zu Innovationen zu entwickeln und \\ senkt das Risiko des Scheiterns. Diese Form der Finanzierung ist ein wesentlicher Bestandteil \\ im globalen Wettbewerb. Der deutsche VC-Markt weist zumindest in der Spätphase der \\ Unterstützung von Start-ups Mängel auf. Gegenüber den USA und China besteht ein \\ erheblicher Aufholbedarf.
}

\begin{abstract}
Unternehmertum ist ein wichtiger Treiber für die wirtschaftliche Entwicklung (Assenza, 2019; Bosma et al., 2018, 483). Junge und innovative Start-ups zeichnen sich durch ein hohes Risiko aus, da sie sich in frühen Unternehmensphasen befinden und meist keine Gewinne und Sicherheiten vorweisen können. Sie unterliegen Einschränkungen bei der Fremdfinanzierung, wenn sie neuartige Geschäftsmodelle verfolgen. Die Finanzierung über Kredite kann an den unterstützenden Aufgaben des Finanzmarkts scheitern, zu denen Risikostreuung und -kontrolle gehören. Venture Capital (VC) füllt diese Finanzierungslücke, durchbricht diese finanziellen Restriktionen und ermöglicht Start-ups, ihre Ideen zu Innovationen weiterzuentwickeln und ihre Produkte und Dienstleistungen zu kommerzialisieren. Diese Finanzierungsform ist

(C) Der/die Autor:in 2021. Open Access: Dieser Artikel wird unter der Creative Commons Namensnennung 4.0 International Lizenz veröffentlicht (creativecommons.org/licenses/by/4.0/deed.de).

Open Access wird durch die ZBW - Leibniz-Informationszentrum Wirtschaft gefördert.
\end{abstract}

Seyer Shafie ist ehemaliger Student der Ostfalia Hochschule für angewandte Wissenschaften.

Matthias Liedtke ist wissenschaftlicher Mitarbeiter
am Entrepreneurship Hub der TU Braunschweig
und der Ostfalia Hochschule.
Prof. Dr. Reza Asghari ist Professor für
Entrepreneurship an der TU Braunschweig
und Ostfalia Hochschule und Leiter des
Entrepreneurship Hub.

Matthias Liedtke ist wissenschaftlicher Mitarbeiter am Entrepreneurship Hub der TU Braunschweig und der Ostfalia Hochschule. 
no Venture Management, die dem US-amerikanischen Modell des Fundraisings folgten und ihre Investitionen auf High-Tech-Start-ups in frühen Unternehmensphasen ausrichteten (Frommann, 2003, 75; Plagge, 2006, 40). Der deutsche Markt trat Anfang der 1990er Jahre in eine Konsolidierungsphase ein, die zu einer Normalisierung der Bewertungsansätze und damit zu sinkenden Start-upWerten führte. Außerdem begannen die Investierenden, Risikodiversifikation und -streuung zu den Hauptkriterien für ihre Portfolios zu bestimmen. Mitte der 1990er Jahre konnte ein Aufschwung in der deutschen VC-Branche verzeichnet werden. Fundraising etablierte sich als der bevorzugte Weg der Kapitalbeschaffung, und es wurde in unabhängige Dachfonds investiert, die teilweise auch bei Institutionen und Versicherungen angesiedelt waren. Große Konzerne gründeten sogenannte CorporateVenture-Capital-Gesellschaften. Außerdem erweiterten neue Technologien, wie Informations- und Kommunikationstechnologien, Biotechnologie und Medizintechnik, die Investitionsziele, die durch neue marktfähige Produkte zu einer Gründungswelle in diesen Bereichen führten. Der deutsche VC-Markt konsolidierte sich in der zweiten Hälfte des Jahres 2000 erneut (Frommann, 2003, 77-82; White Star Capital, 2020, 2).

Die Dot-Com-Blase von 2000 traf den deutschen VCMarkt schwer. Das Vertrauen in die Qualität von Startups in Bezug auf die Geschäftsmodelle sowie auf ihre Kennzahlen und Prognosen sank, sodass VC-Investierende insgesamt risikoscheuer wurden und dies eine Bereinigung in den Portfolios der meisten Venture-Capital-Gesellschaften auslöste. Erst mit dem Start des High-Tech-Gründerfonds (HTGF) entspannte sich die Situation, und der Markt konnte reaktiviert werden (Frommann, 2003, 82; KfW, 2020, 3).

\section{Regierungsprogramme}

Das European Recovery Program (ERP) wird von der Kreditanstalt für Wiederaufbau (KfW) verwaltet und dient der Erweiterung der Eigenkapitalbasis von Existenzgründer:innen. Zu den geförderten Projekten gehören Innovationsaktivitäten wie die Entwicklung und Vermarktung neuer Produkte. Weitere Förderprogramme in Zusammenarbeit mit der KfW sind der ERP-Gründerkredit Universell und ERP Capital (BMWi, o.D. a; Gaida, 2002, 229; Hahn, 2018, 69; KfW, 2020, 1; White Star Capital, 2020, 26).

Der HTGF wurde 2005 als öffentlich-private Partnerschaft zwischen dem Bundesministerium für Wirtschaft und Energie (BMWi), der KfW und sechs Industrieunternehmen gegründet. Das Geschäftsmodell der Start-ups sollte auf einer technologischen Neuentwicklung basieren, die in der Seed-Phase zunächst mit einem Betrag von 1 Mio. Euro ausgestattet werden, der bei einer erfolgreichen Entwicklung auf das Dreifache erhöht werden kann (BMWi, o. D. b; Hahn, 2018, 70-71).

Der EXIST-Forschungstransfer des BMWi gliedert sich in zwei Phasen. Die finanzielle Unterstützung in der ersten Phase deckt Personalkosten und zusätzliche Sachkosten bis zu 250.000 Euro ab. In der zweiten Phase erhalten Start-ups 180.000 Euro in Form eines nicht rückzahlbaren Zuschusses für die Realisierung ihrer technologiegetriebenen Geschäftsidee. Das EXISTGründerstipendium unterstützt Gründer:innen mit einem Stipendium. Im Erfolgsfall werden ein Arbeitsplatz und die notwendige Technik, wie z. B. Labore, zur Verfügung gestellt. Außerdem erhalten die Stipendiat:innen einen monatlichen Zuschuss zu ihren Lebenshaltungskosten in Höhe von 800 Euro bis 2.500 Euo. Zusätzlich können Sachkosten von bis zu 10.000 Euro finanziert werden (Hahn, 2018, 69-70).

Die ERP-EIF-Facility ist eine gemeinsame Anstrengung der deutschen Bundesregierung und des Europäischen Investitionsfonds. Die ERP-EIF-Facility wurde 2004 vom BMWi im Auftrag des deutschen ERP-Sondervermögens eingerichtet und stellt VC mit Priorität für Start-ups in frühen und späteren Phasen bereit. Das Volumen der ERPEIF-Fazilität beträgt 3,2 Mrd. Euro und wird durch das ERP-Sondervermögen finanziert (European Investment Fund, o. D.; White Star Capital, 2020, 26).

\section{Besteuerung und relevante rechtliche Aspekte}

Seit dem 1. Januar 2020 ist das Gesetz zur steuerlichen Forschungsförderung in Kraft, das die Attraktivität des Forschungs- und Innovationsstandorts Deutschland verbessern soll. Es ist ein eigenständiges Steuergesetz, das die privatwirtschaftliche Forschung und Entwicklung fördert. Förderfähig sind Vorhaben in den Bereichen der Grundlagenforschung, der industriellen Forschung und der experimentellen Entwicklung (BMWi, 2020).

2016 hat das Bundeskabinett den „Gesetzesentwurf zur Weiterentwicklung der steuerlichen Verlustverrechnung bei Körperschaften“ beschlossen. Durch diese Gesetzesänderung ist es nun möglich, aufgelaufene Verluste auch dann steuerlich weiterzuverwenden, wenn sich neue Investierende an der Gesellschaft beteiligen. Voraussetzung dafür ist, dass ein Beteiligungserwerb im Sinne des § 8c KStG vorliegt. Zudem unterstützt die INVEST-Venture-Capital-Förderung des BMWi „Business Angel“ (BA) bei der Kontaktaufnahme mit Start-ups in der Frühphase und bei der Bereitstellung von VC. BA erhalten eine steuerfreie Rückzahlung in Höhe von $20 \%$ des Beteiligungs- 
betrags vom Bund (Bundesverband Deutsche Startups, 2016, 2; BMWi, o. D. c, 1-2; Gottschalk et al. (2016).

Der Europäische Gerichtshof hat in seinem Urteil 2015 (C595/13) entschieden, dass die Steuerbefreiung für Fondsverwaltungsleistungen ohne Differenzierung nach dem vom Fonds gehaltenen Vermögen anzuwenden ist. Auf dieser Grundlage ist durch das Investmentsteuerreformgesetz von 2016 der $\S 4$ Nr. 8 lit. h UStG neu gefasst worden. Nach der Erweiterung sind VC-Fonds von der Umsatzsteuer befreit, wenn sie bestimmte Kriterien erfüllen. Hierunter fällt z. B., dass die Anlage des gesammelten Vermögens nach dem Prinzip der Risikostreuung erfolgt oder die Fonds Anteile an mehrere Investierende ausgeben. Alle Kriterien können von VC-Fonds nicht gleichzeitig erfüllt werden, was bedeutet, dass sie weiterhin von der Steuerbefreiung ausgeschlossen sind und Deutschland hierbei als Fondsstandort benachteiligt ist (Ritter, 2019, 125-126; Taxgate, o. D.).

\section{Kulturelle Aspekte}

Im deutschen Wirtschaftssystem werden negative Einflüsse oft überbewertet. Realismus und nicht Idealismus dominiert das Geschäftsklima, innovative Unternehmen werden oft ausgebremst. Die Chancen sind gering, mit einem unausgereiften Businessplan einen Kredit oder VC zu bekommen. Zahlreiche deutsche Erfindungen wurden im Ausland vermarktet, etwa Faxgeräte, das MP3-Format oder Hybridmotoren (KAS, 2011, 8 f.).

Laut Global Entrepreneurship Monitor 2019/2020 lag der Anteil derer, die aktiv an der Gründung oder Führung neuer Unternehmen beteiligt sind (gesamte unternehmerische Frühphase), bei 7,6\%, womit Deutschland in diesem Ranking auf Platz 41 liegt. Zudem gaben 29,7\% der Befragten an, dass die Angst vor dem Scheitern sie davon abhalten würde, den Schritt in die Selbstständigkeit zu wagen, was Deutschland in dieser Kategorie auf Platz 46 bringt (GERA, 2020, 108). Wirtschaftliches Scheitern ist in Deutschland ein Stigma, das den Betroffenen lange anhaftet (Faltin, 2018, 366, 376; KAS, 2011, 8 f.; Remmele et al., 2007, 63).

\section{Der deutsche Venture-Capital-Markt}

Deutschland dominiert die europäische Wirtschaft, ist stark exportorientiert und verfügt über eine robuste Mittelschicht. Deutschlands wachsender VC-Markt basiert zum einen auf seiner robusten Wirtschaft und zum anderen auf dem starken Engagement von Corporate Venture Capital (CVC) und international ausgerichteten Start-ups (White Star Capital, 2020, 2). Die dominierenden VCStandorte sind Berlin, München und Hamburg. Diese
Hubs ziehen aufstrebende Unternehmen an. Berlin ist der Spitzenreiter im deutschen VC-Markt mit über 3.800 Start-ups, 1.200 Fonds und mehr als 3.000 Investitionsrunden und Exits (Dealroom, 2020, 31; EY, 2020, 28; Teare, 2020; White Star Capital, 2020, 21).

Trotz des steigenden Investitionsvolumens waren die VC-Märkte in Großbritannien und Frankreich in den vergangenen drei Jahren durchschnittlich etwa 2,1- und 1,5-mal größer. Dies entspricht einer Differenz des Investitionsvolumens von 700 Mio. bis 1.700 Mio. Euro pro Jahr. Auch die Größe der deutschen VC-Fonds ist in den letzten zehn Jahren mit der Reifung des Marktes stetig gestiegen. In den letzten drei Jahren lag der Durchschnitt bei 154 Mio. Euro. Dies deutet auf eine wachsende Attraktivität des VC-Marktes für inländische Investierende hin. Unter den deutschen Investierenden, die in den letzten zwei Jahren neue Fonds mit einem Volumen von mindestens 100 Mio. Euro aufgelegt haben, waren der HTGF, Deutsche Telekom Capital Partners, Robert Bosch Venture Capital, Linus Capital, Alstin Capital, Acton Capital Partners und BlueYard (EY, 2020, 37; KfW, 2020; White Star Capital, 2020, 29).

\section{Mangel in der Spätphase}

Deutsche Investierende stellen vor allem in der Frühphase VC für Start-ups zur Verfügung, während Wachstums- und Spätphase-Transaktionen von internationalen Fonds dominiert werden. Die solide Kapitalisierung der Frühphase ist vor allem das Ergebnis einer auskömmlich finanzierten und funktionierenden staatlichen Förderung. Die Fondsvolumina der europäischen Fonds sind zu gering für VCInvestitionen in der späteren Phase. Internationale Investierende sind in neun von zehn Runden ab dem niedrigen zweistelligen Millionenbereich vertreten. Für Deutschland steigt damit das Risiko, dass Start-ups und ihre Expertise das Land verlassen. Im Hinblick auf sogenannte Unicorns, Start-ups mit einer Marktbewertung von über $1 \mathrm{Mrd}$. US$\$$, findet sich Deutschland im europäischen Mittelfeld wieder. Unicorns können als Maß für die Fähigkeit von VCMärkten, wiederholt großvolumige Finanzierungsrunden zu verwirklichen, herangezogen werden. In Deutschland existierten 2020 zwölf solcher Unicorns, das sind weniger als in Großbritannien (22), jedoch mehr als in Frankreich (5). Unicorns häufen sich in großen VC-Märkten, global sind deswegen die meisten in den USA und China zu finden (EY, 2020, 37; KfW, 2020; Roland Berger et al., 2017, 19-21; White Star Capital, 2020, 10).

\section{Investitionsbranchen}

Die führenden VC-finanzierten Branchen in Deutschland sind Mobilität, Fintech, B2B-Software, B2C-Anwendun- 
gen und Retail, was hervorhebt, dass in Digitalisierungsund weiteren Zukunftsfeldern ein Rückstand im internationalen Vergleich besteht. In China und den USA stechen in den Transaktionsdaten vor allem Investitionen im Bereich der Mobilen Apps hervor. In Feldern wie Manufacturing oder Robotics, die an klassische Forschungsstärken Deutschlands anknüpfen, hängt der deutsche VC-Markt ebenfalls zurück. In weiteren Technologiebereichen wie Künstliche Intelligenz, Big Data, Clean Technology und Health Tech fallen die VC-Dealvolumen im internationalen Vergleich zwar weniger stark ab, doch ihr relativer Anteil am deutschen VC-Markt ist vergleichsweise gering (KfW, 2020; White Star Capital, 2020, 8).

\section{Handlungsempfehlungen für den deutschen Markt}

In Deutschland erhalten VC-Fonds $5 \%$ ihres Kapitals von Pensionskassen. Im deutschen umlagefinanzierten Rentensystem werden die laufenden Rentenbeiträge direkt an die Rentner:innen weitergegeben und nicht zwischenzeitlich investiert. 2017 verwalteten allein die 1.000 größten Pensionsfonds und Versorgungswerke in Europa einen Kapitalstock von 7 Billionen Euro. Eine vorausschauende Allokation von $0,7 \%$ dieses ruhenden Privatvermögens würde ausreichen, um die VC-Lücke Europas gegenüber den USA zu schließen. Des Weiteren muss die deutsche Bevölkerung die Möglichkeit haben, am Wachstum der digitalen Wirtschaft teilzuhaben, wofür wirtschaftliche Anreize und Sicherheiten erforderlich sind. Dies kann eng verbunden werden mit einer schrittweisen Modernisierung des deutschen Rentensystems. Das Fehlen von groBen Pensionsfonds als VC-Investierende in Deutschland kann nur teilweise kompensiert werden, z. B. durch CVC oder durch die öffentliche Hand mit Initiativen wie HTGF und den Venture-Capital-Fonds für junge Technologieunternehmen Coparion. Eine Möglichkeit wäre, dass der Staat und private Investierende die Beitragszahlenden gegen negative Renditen absichern, wie im Fall der Barmer GEK Fonds oder der NRW-Bank. Diese Fonds sind zu gleichen Teilen durch das Vermögen des ERP und der Partner des VC-Investors Earlybird besichert (Invest Europe, 2020, 20; McKinsey \& Company, 2018, 16; Roland Berger et al., 2017, 9, 33 ff.).

Investitionen in späteren Phasen sind von großer Bedeutung. Nicht alle Start-ups, die in der Frühphase investieren, sind in der Lage, den kostspieligen Markteintritt und einen langfristigen Erfolg zu realisieren. Es ist entscheidend, dass die Unternehmen in der späteren Phase adäquat finanziert werden, sonst können sie sich nicht zu globalen Pionieren entwickeln und bleiben unterfinanzierte Start-ups mit ungenutztem Wachstumspotenzial. Für Deutschland ist es daher wichtig, den Mangel in der Spätphase zu beheben, um Start-ups bessere Wachstums- chancen zu bieten. Die hohe Beteiligung ausländischer Direktinvestierender bei Wachstumsfinanzierungen ist ein Zeichen dafür, dass der deutsche VC-Markt noch nicht ausgereift ist (KfW, 2020, 3; Roland Berger et al., 2017, 16).

Angesichts der zunehmenden Bedeutung digitaler Geschäftsmodelle, die nach ihrer Anschubfinanzierung rasch großvolumige Anschlussfinanzierungen für Wachstum und Marktdurchdringung benötigen, stellt dies ein Problem für die volkswirtschaftliche Zukunftsfähigkeit Deutschlands dar. Die US-Risikokapitalszene hat sich auf die speziellen Bedürfnisse von neugegründeten Unternehmen in der digitalen Wirtschaft eingestellt. In Deutschland muss dieser Entwicklungsschritt erst noch erfolgen. Außerdem sollte ein Zukunftsfonds in Deutschland in Form eines Dachfonds gemeinsam mit der Industrie aufgelegt werden. Ein Dachfonds würde das Risiko für Investierende reduzieren und gleichzeitig die notwendige Größe für signifikante Transaktionen großer institutioneller Investierender, speziell Banken, Versicherungen, Pensionskassen und Stiftungen, ermöglichen (KfW, 2020, 3; Roland Berger et al., 2017, 16).

Außerdem sollte der rechtliche Rahmen Impulse für die Mobilisierung von VC geben. Dieser müsste nicht nur bürokratische und steuerliche Hindernisse beseitigen, z.B. die Mehrwertsteuer auf die Managementgebühr abschaffen, sondern auch Anreize für VC-Investitionen schaffen, z.B. durch Abschreibungsmöglichkeiten oder die Ausweitung des INVEST-Zuschusses auf Investitionen in VCFonds (Roland Berger et al., 2017, 33 ff.).

Auch im Bildungswesen gibt es Ansatzpunkte, die verbessert werden müssen, um die Gründungsmentalität der deutschen Bevölkerung zu verbessern. Die Erfolgsaussichten für „Nerd-Start-ups“, die von Studierenden mit einem starken wissenschaftlichen Hintergrund und ohne Mentor:innen gegründet werden, sind in der Regel aufgrund fehlender betriebswirtschaftlicher Expertise begrenzt. Dies ist ein weiterer Faktor, warum nicht mehr zukunftsweisende Produkte in deutschen Startups entwickelt werden. Folglich ist die Start-up-Szene in Deutschland stark von Betriebswirt:innen dominiert. Durch die Vielzahl der technischen Universitäten ist die naturwissenschaftliche Expertise vorhanden, die stärker mit betriebswirtschaftlichen Inhalten gekoppelt werden muss. Es ist wichtig, ähnlich wie in den USA oder China, das Angebot an Entrepreneurship Education (EE) zu vergrößern und dieses Lehrangebot vor allem mit praktischen Inhalten zu füllen (KAS, 2011, 9). Des Weiteren müssen Studierende die Möglichkeit haben, bereits im Studium auf potenzielle Investierende bzw. Mentor:innen zu treffen, damit EE nicht lediglich als abstraktes Studien- 
fach, sondern als ernsthafte Berufschance gesehen wird. Hierdurch würde nicht nur ein Anreiz für Unternehmertum geschaffen werden. Durch den Erfahrungshorizont von Investierenden oder Unternehmer:innen erhalten Studierende wichtige Anhaltspunkte für die Planung und Umsetzung zukünftiger Geschäftsideen (KAS, 2011, 9).

Die dargestellten Aspekte des deutschen VC-Marktes zeigen, dass auf Ebene der Investitionen und Rahmenbedingungen ein großer Aufholbedarf zu den führenden Ländern wie USA und China besteht. Wenn Deutschland seine Rolle als führende Wirtschaftsnation beibehalten möchte, ist es zwingend notwendig, VC als technologieund innovationsaffiner Finanzierungsform mehr Aufmerksamkeit zu schenken und die gesellschaftlichen und politischen Rahmenbedingungen anzupassen. Eine Lösung, die den Rückstand sofort beseitigen würde, gibt es nicht. Es ist daher wichtig, die hier identifizierten Hemmnisse und Blockaden stückweise abzuschaffen.

\section{Literatur}

Assenza, P. (2016), Identifying fertile ground: Peripheral stakeholder contribution to a healthy entrepreneurial ecosystem, Journal of Business and Entrepreneurship, 28(1), 31.

Bosma, N., M. Sanders und E. Stam (2018), Institutions, entrepreneurship, and economic growth in Europe, Small Business Economics, 51(2), 483-499.

BMWi (o.D. a) Gründungen, Wachstum und Innovationen finanzieren, https://www.bmwi.de/Redaktion/DE/Dossier/mittelstandsfinanzierung. html (17. Juli 2020).

BMWi (o.D. b), High-Tech Gründerfonds, https://www.bmwi.de/Redaktion/DE/Artikel/Mittelstand/gruendungsfinanzierung-high-tech-gruenderfonds.html (17. Juli 2020).

BMWi (2020), Neue Forschungszulage in Deutschland: Deutschland gewinnt als Standort für Forschung und Innovationen weiter an Attraktivität, https://www.bmwi.de/Redaktion/DE/Schlaglichter-derWirtschaftspolitik/2020/02/kapitel-1-10-neue-forschungszulage-indeutschland.html (21. Juli 2020).

Bundesverband Deutsche Startups (2016), Stellungnahme: Gesetzesentwurf zur Weiterentwicklung der steuerlichen Verlustverrechnung bei Körperschaften.

Dealroom (Hrsg.) (2020), Shortage of later stage venture capital in Germany: more acute due to Corona crisis.

EY (Hrsg.) (2020), Growth with purpose: German Tech start-ups changing society: Venture capital and start-ups in Germany 2019.
European Investment Fund (o.D.), The ERP-EIF Facility, https://www.eif. org/what_we_do/resources/erp/index.htm (15. September 2021).

Faltin, G. (Hrsg.) (2018), Handbuch Entrepreneurship, 365-381, Springer Gabler.

Gaida, M. (2002), Venture Capital in Deutschland und den USA: Finanzierung von Start-ups im Gefüge von Staat, Banken und Börse, Springer.

Gantenbein, P., A. Kind und C. Volonté (2019), Individualism and Venture Capital: A Cross-Country Study, Management International Review, 59(5), 741-777.

GERA (Global Entrepreneurship Research Association) (Hrsg.) (2020), Global Entrepreneurship Monitor 2019/2020.

Gottschalk, S., Egeln, J., Herrmann, F., Hupperts, S., Reuß, K., Köhler, M., Bersch, J., Wagner, S. (2016), Evaluation des Förderprogramms „INVEST-Zuschuss für Wagniskapital“, ZEW-Gutachten und Forschungsberichte.

Hahn, C. (Hrsg.) (2018), Finanzierung von Start-up-Unternehmen: Praxisbuch für erfolgreiche Gründer: Finanzierung, Besteuerung, Investor Relations, Springer Gabler.

Invest Europe (Hrsg.) (2020), Investing in Europe: Private Equity Activity 2019.

Frommann, H. (2003), Venture Capital und Private Equity in Deutschland: Historie - Gegenwart - Zukunft, E-Venture-Management, 73-86, Gabler.

KAS (Konrad Adenauer Stiftung) (Hrsg.) (2011), Start-ups in Deutschland und den USA (Report). Analysen und Argumente.

KfW (2020), KfW Venture Capital Studie 2020: VC-Markt in Deutschland: Reif für den Entwicklungsschritt.

McKinsey \& Company (Hrsg.) (2018), Tech-Titanen made in Germany. Eine Perspektive.

Plagge, A. (2006), Public Policy for Venture Capital, 27-95.

Remmele, B., M. Schmette und G. Seeber (Hrsg.) (2007), Educating Entrepreneurship: Didaktische Ansätze und europäische Perspektiven - Didactical Approaches and European Perspectives, DUV.

Ritter, F. (2019), Die Abgrenzung der privaten Vermögensverwaltung von der Gewerblichkeit bei Private Equity-Fonds, 121-130.

Roland Berger, International Economy Foundation, Bundesverband Deutscher Beteiligungsgesellschaften (Hrsg.) (2017), Treibstoff Venture Capital: Wie wir Innovation und Wachstum befeuern.

Schaal, A., M. et al. (Hrsg.) (2010), Die Relevanz von Venture Capital für Innovation und Beschäftigung: Theoretische Klärung und empirische Analyse, 63, Peter Lang.

Sifted (o.D.), https://sifted.eu/rankings/german-startups-top-rankings (15. September 2021)

Taxgate (o.D.), EuGH-Urteil zur Umsatzsteuerbefreiung für die Fondsverwaltung.

Teare, G. (2020), European Venture Picks Up In Q3 Following Slow Start, 20. Oktober, https://news.crunchbase.com/news/europe-vc-fundingreport-q3-2020/ (15. September 2021).

Telgheder (2019), Deutschland riskiert die Abwanderung von Know-how im Biotechbereich, https://www.handelsblatt.com/meinung/kommentare/kommentar-deutschland-riskiert-die-abwanderung-vonknow-how-im-biotechbereich/24571710.html (22. September 2020).

White Star Capital (Hrsg.) (2020), German Venture Capital Landscape 2020: From the eyes of an International Investor (Report).

Title: German Venture Capital Market - Investments and Framework

Abstract: Innovative start-ups are characterised by a high level of risk for several reasons. Venture capital (VC) enables start-ups to develop their ideas into innovations and lowers the risk of failure. This form of financing is an essential component in the global competition. This paper aims at examining the German VC market with regard to its investments and framework conditions. The framework conditions that influence VC transactions are, on one end of the spectrum, the political and regulatory environment and, on the other, the innovation climate such as societal attitudes towards entrepreneurial and opportunity risk. The paper concludes with some important policy implications. 